\title{
Neutrophil to lymphocyte ratio, a biomarker in non-muscle invasive bladder cancer: a single-institutional longitudinal study
}

\author{
Vincenzo Favilla ${ }^{1}$, Tommaso Castelli ${ }^{1}$, Daniele Urzì ${ }^{1}$, Giulio Reale ${ }^{1}$, Salvatore Privitera ${ }^{1}$, Antonio Salici ${ }^{1}$, \\ Giorgio Ivan Russo ${ }^{1}$, Sebastiano Cimino ${ }^{1}$, Giuseppe Morgia ${ }^{1}$
}

1 Sezione Urologia, Dipartimento di Chirurgia, Università di Catania, Italia

\section{ABSTRACT}

Background: Bladder cancer represents one of the most important clinical challenges in urologic practice. In this context, inflammation has an important role in the development and progression of many malignancies. The objective of the present study was to evaluate the prognostic value of pre-treatment Neutrophil to lymphocyte ratio (NLR) on the risk of recurrence and progression in patients with primary non-muscle invasive bladder cancer.

Materials and Methods: Data obtained from 178 bladder cancer patients who underwent transurethral resection of bladder tumor (TURB) between July 2008 and December 2014 were evaluated prospectively. NLR was obtained from each patient before TURB and defined as the absolute neutrophil count divided by the absolute lymphocyte count. Cox proportional hazards regression model was performed to calculate disease recurrence and progression including NLR.

Results: During the follow-up study (median: 53 months), 14 (23.3\%) and 44 (37.9\%) $(\mathrm{p}=0.04)$ patients respectively with NLR $<3$ and $\geq 3$ experienced recurrence and $2(3.3 \%)$ and $14(11.9 \%)$ experienced progression $(p=0.06)$, respectively. At the multivariate Cox regression analysis, NLR $\geq 3$ was associated with worse disease recurrence (HR: 2.84; $\mathrm{p}<0.01)$. No association was found regarding disease progression. The 5-year recurrence free survival was $49 \%$ and $62 \%$ in patients with NLR $\geq 3$ and $<3 \quad(p<0.01)$. The 5 -year progression free survival was $77 \%$ and $93 \%$ in patients with NLR $\geq 3$ and $<3$ $(\mathrm{p}=0.69)$.

Conclusion: NLR predicts disease recurrence but not disease progression in NMIBC patients. NLR alterations may depend of tumor inflammatory microenvironment.

\section{ARTICLE INFO}

\section{Keywords:}

Urinary Bladder Neoplasms;

Neutrophils; Biomarkers

Int Braz J Urol. 2016; 42: 685-93

Submitted for publication:

May 01, 2015

Accepted after revision:

October 26, 2015

\section{INTRODUCTION}

Bladder cancer represents one of the most important clinical challenges in urologic practice. At the time of initial diagnosis, approximately $70 \%$ of patients have cancers confined to the epithelium or the subepithelial connective tissue. In general, these cancers are primarily managed by endoscopic resection (TURB) (1-3). The dilemma in the management of non-muscle-invasive bladder cancer (NMIBC) still remain the risk of recurrence ranging from 30\% up to nearly $80 \%$ and depending on the risk profile, up to $45 \%$ of tumors may progress to muscle-invasive disease within 5 years after initial diagnosis (4). To manage patients with NMIBC based on their individual risk, based 
on the course of the disease in well-controlled prospective randomized clinical trials, the European Organization for Research and Treatment of Cancer (EORTC) has developed risk tables to predict the individual risks for tumor recurrence or progression to muscle-invasive disease (4). According to the EORTC risk table, using a scoring system based on previous recurrence rate, tumor number, tumor diameter, T category, World Health Organization (WHO) grade, and the presence of concurrent carcinoma in situ (CIS), to estimate the risk of disease recurrence and progression at 1 and 5 years, patients with bladder cancer were stratified into low-, intermediate-, and high-risk group, which may guide clinical management (5). To further improve the predictive accuracy of risk Tables, a large number of clinical, molecular, biological, and environmental factors are available that have been studied in relation to bladder cancer development, recurrence, and/or progression in NMIBC.

In this context, inflammation has an important role in the development and progression of many malignancies (6). Putative mechanisms include the increased supply of factors that promote carcinogenesis and tumor progression by cells of the innate immune systems such as neutrophils and decreased anti-tumor response by immune cells of the adaptive system such as lymphocytes (7-11).

Moreover, the neutrophil to-lymphocyte ratio (NLR), which can easily be calculated from routine complete blood counts (CBCs) with differentials, is an emerging marker of host inflammation and it has been shown to be an independent prognostic factor for a variety of solid malignancies, including the urinary tract (12-14). Although a recent study found that preoperative NLR was associated with advanced pathologic stage at the time of cystectomy, as well as increased risk for disease recurrence, cancer-specific mortality and all-cause mortality (15), there are sparse and retrospective data on the prognostic role of NLR in patients with NMIBC. The purpose of our study was to evaluate the prognostic value of pre-treatment NLR on the risk of recurrence and progression in patients undergoing TURB for primary NMIBC.

\section{MATERIALS AND METHODS}

Data obtained from 178 bladder cancer patients who underwent transurethral resection of bladder tumor (TURB) between July 2008 and December 2014 were evaluated prospectively after institutional internal review board approval was obtained. The diagnosis of bladder cancer was histologically confirmed by TURB in each patient. The clinical $\mathrm{T}$ stage of a bladder tumor was determined according to the 2002 Union International Contre le Cancer (UICC) TNM classification of bladder tumors. Tumor size was defined as the maximum tumor dimension estimated at the time of TURB and/or by clinical imaging. Tumors size were categorized in one group if its size was above $3 \mathrm{~cm}$ and into another if below $3 \mathrm{~cm}$. The number and shape of the tumors were examined in the same manner. Concomitance of CIS was revealed in the surgical pathology of TURB. According to the pathology reports, patients were grouped as non-muscle invasive bladder cancer (NMIBC) or muscle-invasive bladder cancer (MIBC). Only patients with NMIBC were included in our study. Demographics and laboratory data, including hemoglobin ( $\mathrm{Hb}$ ) levels, platelet count, neutrophil count, lymphocyte count and serum values of neutrophil to lymphocyte ratio (NLR) were obtained from each patient before TURB. The NLR was defined as the absolute neutrophil count divided by the absolute lymphocyte count. Patient demographics, preoperative full blood count, operative details, and standard histologic tumor characteristics were recorded. Exclusion criteria for the present study were previous operation due to bladder tumor, ongoing treatment for bladder cancer, hematologic disorders or history of conditions that may have influenced blood cell lines such as connective tissue disease, presence of an active infection and/or immunodeficiency virus infection at the time of surgical intervention, prior or concomitant intra-vesical therapy with Bacille Calmette-Guérin (BCG), prior blood transfusion, and the presence of other cancer types or prior chemotherapy. Patients with non-urothelial cancer or for primary prostatic urothelial carcinoma were also excluded in order to maintain a homogenous cohort. 
A second TURB was routinely performed in patients who had a T1 or high-grade tumor on initial TURB. Patients received post-operative intra-vesical instillations based on tumor characteristics, and at the discretion of the treating urologist. Postoperative follow-up consisted of cystoscopy and upper urinary tract imaging performed every three months for the first 2 years, every 6 months 2 to 5 years after surgery, and annually thereafter. Patients with a suspected recurrence underwent TURB. Disease recurrence was defined as the first pathologically confirmed tumor relapse in the bladder, regardless of the tumor stage. Disease progression was defined according to the International Bladder Cancer Group consensus definition for progression in NMIBC, in the presence of an increase in T category from CIS or Ta to T1 (lamina propria invasion), development of $\geq \mathrm{T} 2$ or lymph node $(\mathrm{N}+)$ disease or distant metastasis (M1), or an increase in grade from low to high.

All participants provided written informed consent before enrolment and the study was conducted in accordance with regulatory standards of Good Clinical Practice and the Declaration of Helsinki (1996). The study was approved by our Institutional Research Ethics Committee.

\section{Statistical analysis}

All statistical analyses were completed using SPSS versus 19 software (SPSS Inc, IBM Corp, Somers, NY, USA). The qualitative data were tested using the chi-square test or Fisher's exact test as appropriate and the continuous variables, presented as median, were tested by Mann-Whitney U-test.

The significance of the clinic and pathological variables associated with disease recurrence and progression were assessed using the Cox proportional hazards regression model, including age, stage, grade, tumor size, focality, BMI, gender, diabetes, Cis and NLR. Based on the ROC curve, we used a cut-off of 3 for the NLR with the best balance between sensitivity (50\%) and specificity (76\%) (area under the curve 0.60, 95\% confidence interval [CI] 0.51-0.69; $\mathrm{p}<0.05$ ).

Curves were tested with the log-rank test. Predictive accuracy of the model was assessed in term of the area under the curve (AUC) value, incorporating all significant and independent predictors. AUC values were also calculated by applying base model to the study cohort. The areas under the curve were compared via the Mantel-Haenszel test. For all statistical comparisons significance was considered as $p<0.05$. One thousand bootstrap resamples were used for all accuracy estimates and to reduce overfit bias. $\mathrm{P}$-value $<0.05$ was considered as an indicator of statistical significance.

\section{RESULTS}

Table- 1 lists the baseline characteristics of the cohort. This study included 148 (83.1\%) male and $30(16.9 \%)$ female patients. The median age of all 178 patients enrolled in the study was 69.27 (IQR: 63.78-79.44), with a median follow-up of 53 months (IQR: 33.0-76.25). Patients with NLR $\geq 3$ were older (74.45 versus 67.94; $p=0.02)$ and exhibited significant differences in term of pathological stage $(26.6 \%$ versus $20.33 \%$; $p<0.05)$, number of multifocal tumors (53.4\% versus $23.72 ; \mathrm{p}=0.04$ ) and $\mathrm{Cis}(50.0 \%$ versus $16.94 \% ; \mathrm{p}<0.05)$ if compared with those with NLR<3 (Table-2).

During the follow-up study, 14 (23.3\%) and $44(37.9 \%)(p=0.04)$ patients respectively with NLR $<3$ and $\geq 3$ experienced recurrence and $2(3.3 \%)$ and 14 (11.9\%) experienced progression $(\mathrm{p}=0.06)$, respectively. At the multivariate Cox regression analysis, NLR $\geq 3$ was associated with worse disease recurrence (HR: 2.84 [IQR: 1.505.75]; $\mathrm{p}<0.01)$. Pathological stage pT1 $(\mathrm{p}<0.01)$, high grade $(p<0.01)$, no. of tumors $(p<0.01)$ and smoking status $(p<0.01)$ were independently predictors of disease recurrence. No association was found between $\mathrm{NLR} \geq 3$ and disease progression at the multivariate Cox regression analysis (Table-3). The 5-year recurrence free survival was $49 \%$ and $62 \%$ in patients with NLR $\geq 3$ and $<3$ $(\mathrm{p}<0.01)$ (Figure-1). The 5-year progression free survival was $77 \%$ and $93 \%$ in patients with $\mathrm{NLR} \geq 3$ and $<3(\mathrm{p}=0.69)$.

The bootstrapping calculations generally confirmed the $p$-values of the conventional Cox-regression analysis with larger ranges of 95\% CI of the ORs (data not shown). After one thousand 
Table 1 - Baseline characteristics of the patients.

\begin{tabular}{|c|c|}
\hline & Total $(n=178)$ \\
\hline Age, year, median (IQR) & $69.27(63.78-79.44)$ \\
\hline \multicolumn{2}{|l|}{ Gender, no. (\%) } \\
\hline Female & $30(16.9)$ \\
\hline Male & $148(83.1)$ \\
\hline BMI, median (IQR) & $27.29(24.2-29.4)$ \\
\hline Hypertension, no. (\%) & $108(60.7)$ \\
\hline Diabetes, no. (\%) & $54(30.3)$ \\
\hline Dyslipidemia, no. (\%) & $36(20.2)$ \\
\hline NLR, median (IQR) & $2.41(1.69-3.62)$ \\
\hline \multicolumn{2}{|l|}{ Pathologic stage, no. (\%) } \\
\hline pTa & $138(77.5)$ \\
\hline pT1 & $40(22.5)$ \\
\hline \multicolumn{2}{|l|}{ Pathologic grade, no. (\%) } \\
\hline Low grade & $126(70.8)$ \\
\hline High grade & $40(22.5)$ \\
\hline \multicolumn{2}{|l|}{ Concomitant CIS, no. (\%) } \\
\hline No & $128(71.9)$ \\
\hline Yes & $50(28.1)$ \\
\hline \multicolumn{2}{|l|}{ No. of tumours (\%) } \\
\hline 1 & $118(66.3)$ \\
\hline $2-7$ & $54(30.3)$ \\
\hline$\geq 8$ & $6(3.4)$ \\
\hline \multicolumn{2}{|l|}{ Tumour size, cm, no. (\%) } \\
\hline$<3 \mathrm{~cm}$ & $96(53.9)$ \\
\hline$\geq 3 \mathrm{~cm}$ & $82(46.1)$ \\
\hline \multicolumn{2}{|l|}{ Smoking status } \\
\hline Never & $18(10.1)$ \\
\hline Former & $74(41.6)$ \\
\hline Current & $86(48.3)$ \\
\hline \multicolumn{2}{|l|}{ EORTC Recurrence } \\
\hline Low & $48(27.0)$ \\
\hline Intermediate & $126(70.8)$ \\
\hline High & $4(2.2)$ \\
\hline \multicolumn{2}{|l|}{ EORTC Progression } \\
\hline Low & $58(32.6)$ \\
\hline Intermediate & $82(46.1)$ \\
\hline High & $38(21.3)$ \\
\hline
\end{tabular}

$\mathbf{I Q R}$ = interquartile range; $\mathbf{B M I}$ = body mass index; $\mathbf{N L R}=$ neutrofil-to-lymphocyte ratio; $\mathbf{C I S}=$ carcinoma in situ bootstrapping resampling, the derived ROC of the base model was 0.75 , while when incorporating the NRL to this model the derived AUC value resulted in 0.78 . However, the gain in accuracy (3\%) was not statistical significant.

\section{DISCUSSION}

Non-muscle invasive bladder cancer (NMI$\mathrm{BC}$ ) represents a heterogeneous group of tumors with different rates of recurrence, progression, and disease-related mortality. NMIBC are initially treated with TURB after which adjuvant therapy should be considered according to tumor-based risk stratification that helps identify the appropriate treatment for each group of patients based on their risk for recurrence or progression (3). To further improve the ability to select the appropriate treatment for each individual patient, especially in doubtful cases such as patients at intermediate or high risk for recurrence or progression, additional, independent pretreatment predictors of outcome may help to further individualize treatment options within each risk group $(5,16)$. In recent years, the host inflammatory response has gained increasing attention in oncology research. In fact, increasing evidence showed the association of inflammation and cancer. While initially thought to represent an anti-tumor response, immune cells, particularly those of the innate immune system, also exhibit effects that promote carcinogenesis and cancer progression. Proposed mechanisms include increased supply of growth factors, survival factors, pro-angiogenic factors, extracellular matrix-modifying enzymes and inductive signals that may lead to epithelial-to-mesenchymal transition $(7,17)$. Recently, several inflammatory parameters obtained from blood tests, including C-reactive protein, NLR, platelet-lymphocyte ratio, and albumin levels, were associated with the treatment outcome of several malignancies (18). In this context, there is a biological rationale for using NLR, the ratio of circulating neutrophils (immune cells of the innate system) to lymphocytes (immune cells of the adaptive system), as a measure of the systemic host response when evaluating the association between inflammation and cancer outcomes. In fact, an enhanced neutrophil 
Table 2 - Clinical characteristics of the patients according to neutrophil-to-lymphocyte ratio.

\begin{tabular}{|c|c|c|c|}
\hline & NLR $<3(n=118)$ & $N L R \geq 3(n=60)$ & $p$-value \\
\hline Age, year, median (IQR) & $67.94(63.33-76.97)$ & 74.45 (44.75-80.41) & 0.02 \\
\hline Gender, no. (\%) & & & 0.96 \\
\hline Female & $20(16.9)$ & $10(16.7)$ & \\
\hline Male & $98(83.1)$ & $50(83.3)$ & \\
\hline BMI, median (IQR) & $27.29(24.22-32.44)$ & $27.15(24.20-28.39)$ & 0.44 \\
\hline Hypertension, no. (\%) & $70(59.3)$ & $38(63.3)$ & 0.60 \\
\hline Diabetes, no. (\%) & $32(27.1)$ & $22(36.7)$ & 0.19 \\
\hline Dyslipidemia, no. (\%) & $22(18.6)$ & $14(23.3)$ & 0.46 \\
\hline NLR, median (IQR) & $1.94(1.57-2.41)$ & $4.02(3.55-4.82)$ & $<0.01$ \\
\hline Pathologic stage, no. (\%) & & & 0.03 \\
\hline pTa & $103(87.28)$ & $44(73.3)$ & \\
\hline pT1 & $15(12.72)$ & $16(26.6)$ & \\
\hline Pathologic grade, no. (\%) & & & 0.22 \\
\hline Low grade & $90(76.27)$ & $36(60.0)$ & \\
\hline High grade & $28(23.73)$ & $24(40.0)$ & \\
\hline Concomitant CIS, no. (\%) & & & 0.01 \\
\hline No & $98(83.05)$ & $24(40.0)$ & \\
\hline Yes & $20(16.94)$ & $36(60.0)$ & \\
\hline No. of tumours (\%) & & & 0.04 \\
\hline 1 & $90(76.28)$ & $28(46.6)$ & \\
\hline $2-7$ & $28(23.72)$ & $26(43.4)$ & \\
\hline$\geq 8$ & $0(0)$ & $6(10.0)$ & \\
\hline Tumour size, cm, no. (\%) & & & 0.60 \\
\hline$<3 \mathrm{~cm}$ & $67(56.78)$ & $29(48.33)$ & \\
\hline$\geq 3 \mathrm{~cm}$ & $51(43.22)$ & $31(51.66)$ & \\
\hline Smoking status & & & 0.06 \\
\hline Never & $12(10.2)$ & $6(10.0)$ & \\
\hline Former & $63(53.4)$ & $11(18.3)$ & \\
\hline Current & $43(36.4)$ & $43(71.7)$ & \\
\hline EORTC Recurrence & & & 0.34 \\
\hline Low & $32(27.1)$ & $16(26.7)$ & \\
\hline Intermediate & $86(72.9)$ & $40(66.6)$ & \\
\hline High & $0(0.0)$ & $4(6.7)$ & \\
\hline EORTC Progression & & & 0.15 \\
\hline Low & $39(33.0)$ & $19(31.7)$ & \\
\hline Intermediate & $62(52.5)$ & $20(33.3)$ & \\
\hline High & $17(14.5)$ & $21(35.0)$ & \\
\hline Chemotherapy instillation & 90 (76.27) & $40(66.6)$ & 0.17 \\
\hline Immunotherapy instillation & $10(8.5)$ & $9(15.0)$ & 0.18 \\
\hline
\end{tabular}

$\mathbf{I Q R}=$ interquartile range; $\mathbf{B M I}$ = body mass index; $\mathbf{N L R}=$ neutrofil-to-lymphocyte ratio; $\mathbf{C I S}=$ carcinoma in situ 
Table 3 - Multivariate Cox-regression analysis for predictors of disease recurrence and progression adjusted for BMI and intravesical therapy.

\begin{tabular}{|c|c|c|c|c|}
\hline & \multicolumn{2}{|c|}{ Disease Recurrence } & \multicolumn{2}{|c|}{ Disease Progression } \\
\hline & $\mathrm{HR}(95 \% \mathrm{Cl})$ & $p$-value & $\mathrm{HR}(95 \% \mathrm{CI})$ & $p$-value \\
\hline Age, year & & & $1.02(0.94-1.11)$ & 0.60 \\
\hline Gender, female vs. male & $0.48(0.15-1.53)$ & 0.22 & $0.30(0.10-0.50)$ & 0.98 \\
\hline Diabetes, yes vs. no & $1.25(0.64-2.42)$ & 0.50 & $1.20(0.19-7.54)$ & 0.84 \\
\hline \multicolumn{5}{|l|}{ Pathologic stage } \\
\hline $\mathrm{pTa}$ & 1.00 (Ref.) & & 1.00 (Ref.) & \\
\hline pT1 & $4.18(1.87-9.35)$ & $<0.01$ & $5.75(0.54-60.99)$ & $<0.05$ \\
\hline \multicolumn{5}{|l|}{ Pathologic grade } \\
\hline Low grade & 1.00 (Ref.) & & 1.00 (Ref.) & \\
\hline High grade & $2.88(1.33-6.18)$ & $<0.01$ & $2.96(0.46-18.86$ & $<0.05$ \\
\hline \multicolumn{5}{|l|}{ Concomitant CIS } \\
\hline No & 1.00 (Ref.) & & 1.00 (Ref.) & \\
\hline Yes & $2.20(0.82-5.88)$ & 0.11 & $7.27(1.01-14-76)$ & $<0.05$ \\
\hline \multicolumn{5}{|l|}{ No. of tumours } \\
\hline 1 & 1.00 (Ref.) & & 1.00 (Ref.) & \\
\hline $2-7$ & $2.25(1.25-4.07)$ & $<0.01$ & $5.33(0.69-41.25)$ & 0.11 \\
\hline$\geq 8$ & $1.62(0.67-7.12)$ & $<0.01$ & $0.98(0.60-1.20)$ & 0.98 \\
\hline \multicolumn{5}{|l|}{ Tumour size, cm } \\
\hline$<3 \mathrm{~cm}$ & 1.00 (Ref.) & & 1.00 (Ref.) & \\
\hline$\geq 3 \mathrm{~cm}$ & $1.57(0.84-2.92)$ & 0.15 & $2.26(0.42-12.27)$ & 0.34 \\
\hline \multicolumn{5}{|l|}{ Smoking status } \\
\hline Never & 1.00 (Ref.) & & 1.00 (Ref.) & \\
\hline Former & $3.13(1.20-8.78)$ & $<0.01$ & $1.14(0.30-3.04)$ & $<0.05$ \\
\hline Current & $1.78(0.95-3.30)$ & $<0.01$ & $5.62(1.32-9.45)$ & $<0.05$ \\
\hline \multicolumn{5}{|l|}{ NLR } \\
\hline$<3$ & 1.00 (Ref) & & 1.00 (Ref.) & \\
\hline$\geq 3$ & $2.84(1.50-5.75)$ & $<0.01$ & $5.35(0.39-73.70)$ & 0.21 \\
\hline
\end{tabular}

response and/or suppression of lymphocyte leading to a high NLR might promote carcinogenesis and inhibit anti-tumor immune response (18, 19). Several studies have shown that a pretreatment high NLR was associated with worse disease-specific and overall survival in muscle-invasive bladder cancer and the upper urinary tract $(14,15,18,20)$. Similarly, a correlation between high NLR levels and muscle-invasive disease at TURB was found by other studies $(2,18)$. The role of negative predictor of recurrence-free survival and cancer specific survival of elevated NLR was also confirmed in a recent meta-analysis including 17 studies involving 3159 cases with urinary cancers (21). However, most previous studies which evaluated the predictive value of NLR in bladder cancer, were retrospective and included a mixed and heterogeneous group of patients with muscle-invasive and high risk non-muscle invasive tumors. Besides heterogeneity, divergence may result from many other factors, including age distribution, gender, lifestyle and so on. Herein we found an association between high NLR levels and worse disease recurrence-free survival even after 
adjustment for common risk factors but not for progression free-survival as recently reported by a retrospective study (18). These findings are consistent with previous reports that found an association between greater NLR and unfavorable tumor characteristics, worse recurrence-free, disease specific and overall survival in patients with muscle-invasive bladder cancer or high-risk patients with NMIBC $(2,18)$. However, on the contrary to recent report (18), our study did not found association between higher NLR and disease progression at the multivariate analysis. We recognize that our study is limited by its small sample size and non-randomized nature. Furthermore, we acknowledge the relative arbitrary cut point of NLR ratio used for the analyses in our study, nevertheless, this threshold allows our data to be contextualized in light of previously published analyses, which, likewise, dichotomized NLR $(2,7,15)$. In addition, despite the use of standard treatment protocols, information regarding the use of intra-vesical maintenance treatment, which may have influenced outcomes, was lacking. Furthermore, Ta and T1 category tumors are distinct diseases that may be associated in a different manner with NLR. Larger cohorts are required to evaluate NLR separately in both these groups. Finally, we recognize that these data are from a single, tertiary referral institution and, as such, require external validation. Nevertheless, within the limitations of a nearly-phase study for marker assessment, our findings suggest that NLR is a potential prognostic marker for prediction of disease recurrence in NMIBC and may better risk-stratify patients in the pre and postoperative settings in order to guide treatment strategies.

It could be also postulated that NLR alterations in NIMBC patients mainly depends of tumor inflammatory microenvironment and cancer biology raising new opportunities for therapeutic interventions (22). The near future of NLR application could be also directed into the interpretation of immune response of BCG therapy. It should be in fact taken into account that NLR is an expression of the immune system, a marker that can be also associated with immunotherapy and considered as a potential predictive factor of BCG response.

Figure 1 - Kaplan-Meier estimates of recurrence-free survival stratified by NLR.

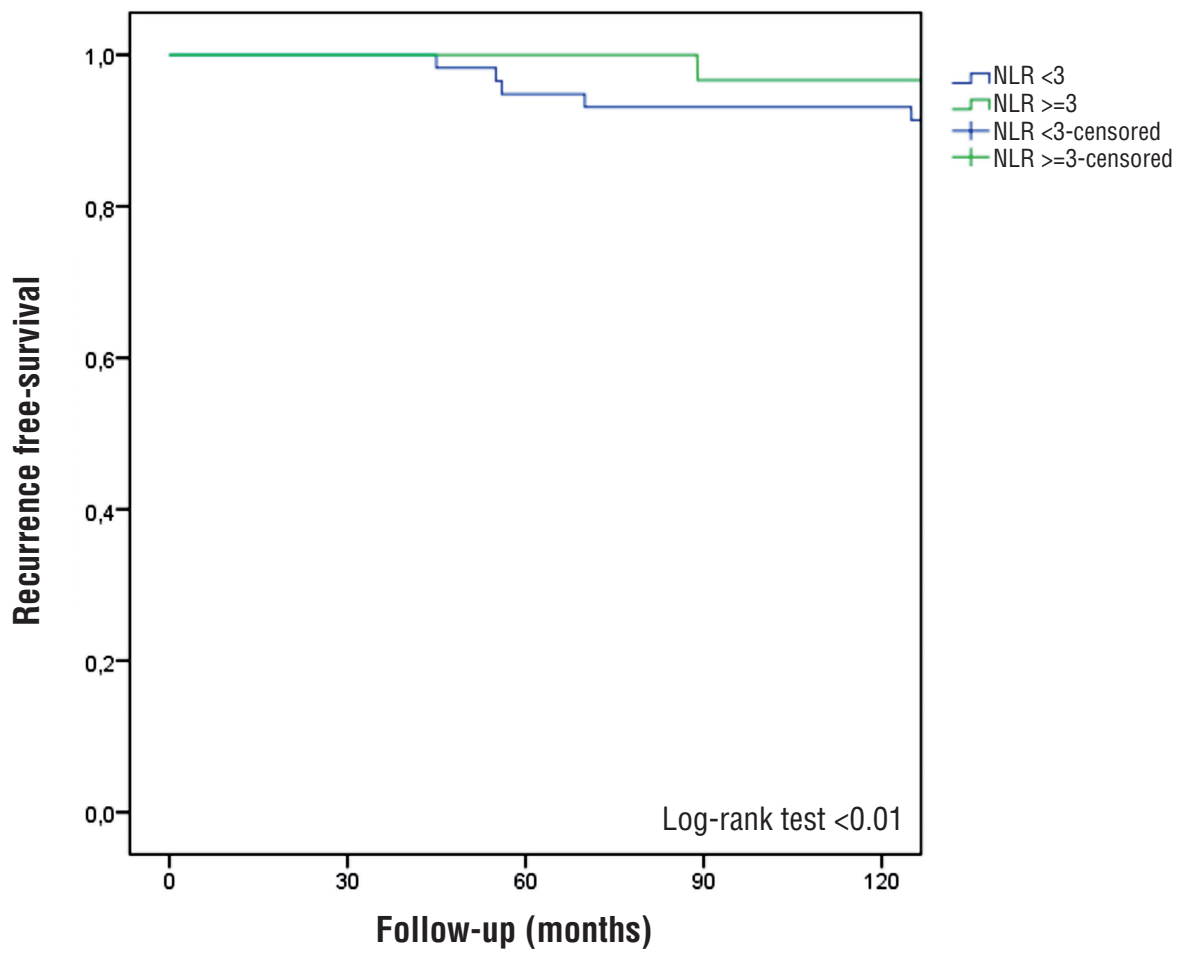


Further prospective, well-controlled clinical studies of diverse patients in multiple institutions are required to validate the role of NLR as a prognostic marker, which may improve current risk stratification tools and treatment outcome in this group of patients.

\section{CONCLUSIONS}

In conclusion, NLR is an inexpensive hematologic test based on commonly measured parameters that may predict disease recurrence, $\mathrm{T} 1$ category, concomitant Cis and high tumor grade in a cohort of patients with NMIBC. Whereas our results suggest that NLR may have a role as a prognostic biomarker, further studies are needed to maximize the clinical utility of NLR in patients with NMIBC.

\section{ABBREVIATIONS}

NLR = Neutrophil to lymphocyte ratio NMIBC $=$ Non muscle invasive bladder cancer TURB $=$ Transurethral resection of bladder tumor AUC $=$ Area under the curve

\section{CONFLICT OF INTEREST}

None declared.

\section{REFERENCES}

1. Gkritsios P, Hatzimouratidis $K$, Kazantzidis S, Dimitriadis G, Ioannidis E, Katsikas V. Hexaminolevulinate-guided transurethral resection of non-muscle-invasive bladder cancer does not reduce the recurrence rates after a 2-year follow-up: a prospective randomized trial. Int Urol Nephrol.2014;46:927-33.

2. Can C, Baseskioglu B, Yılmaz M, Colak E, Ozen A, Yenilmez A. Pretreatment parameters obtained from peripheral blood sample predicts invasiveness of bladder carcinoma. Urol Int.2012;89:468-72.

3. Babjuk M, Burger M, Zigeuner R, Shariat SF, van Rhijn BW, Compérat $E$, et al. EAU guidelines on non-muscle-invasive urothelial carcinoma of the bladder: update 2013. Eur Urol.2013;64:639-53.
4. Sylvester RJ, van der Meijden AP, Oosterlinck W, Witjes JA, Bouffioux C, Denis $L$, et al. Predicting recurrence and progression in individual patients with stage $\mathrm{Ta} T 1$ bladder cancer using EORTC risk tables: a combined analysis of 2596 patients from seven EORTC trials. Eur Urol.2006;49:466-5.

5. van Rhijn BW, Zuiverloon TC, Vis AN, Radvanyi F, van Leenders GJ, Ooms BC, et al. Molecular grade (FGFR3/MIB1) and EORTC risk scores are predictive in primary nonmuscle-invasive bladder cancer. Eur Urol.2010;58:433-41.

6. Grivennikov SI, Greten FR, Karin M. Immunity, inflammation, and cancer. Cell.2010;140:883-99.

7. Hanahan D, Weinberg RA. Hallmarks of cancer: the next generation. Cell.2011;144:646-74.

8. Kaynar M, Yıldırım ME, Badem H, Caviş M, Tekinarslan $\mathrm{E}$, Istanbulluoğlu MO, et al. Bladder cancer invasion predictability based on preoperative neutrophil-lymphocyte ratio. Tumour Biol.2014;35:6601-5.

9. Dip N, Reis ST, Abe DK, Viana NI, Morais DR, Moura CM, et al. Micro RNA expression and prognosis in low-grade noninvasive urothelial carcinoma. Int Braz J Urol.2014;40:644-9.

10. Dobbs RW, Hugar LA, Revenig LM, Al-Qassab S, Petros JA, Ritenour CW, et al. Incidence and clinical characteristics of lower urinary tract symptoms as a presenting symptom for patients with newly diagnosed bladder cancer. Int Braz J Urol.2014;40:198-203.

11. Zhao J, Dong D, Sun L, Zhang G, Sun L. Prognostic significance of the epithelial-to-mesenchymal transition markers e-cadherin, vimentin and twist in bladder cancer. Int Braz J Urol.2014;40:179-89.

12. Proctor MJ, McMillan DC, Morrison DS, Fletcher CD, Horgan PG, Clarke SJ. A derived neutrophil to lymphocyte ratio predicts survival in patients with cancer. $\mathrm{Br} J$ Cancer.2012;107:695-9.

13. Guthrie GJ, Charles KA, Roxburgh CS, Horgan PG, McMillan DC, Clarke SJ. The systemic inflammation-based neutrophillymphocyte ratio: experience in patients with cancer. Crit Rev Oncol Hematol.2013;88:218-30.

14. Templeton AJ, McNamara MG, Šeruga B, Vera-Badillo FE, Aneja P, Ocaña A, et al. Prognostic role of neutrophil-tolymphocyte ratio in solid tumors: a systematic review and meta-analysis. J Natl Cancer Inst.2014;106:dju124.

15. Viers BR, Boorjian SA, Frank I, Tarrell RF, Thapa P, Karnes RJ, et al. Pretreatment neutrophil-to-lymphocyte ratio is associated with advanced pathologic tumor stage and increased cancer-specific mortality among patients with urothelial carcinoma of the bladder undergoing radical cystectomy. Eur Urol.2014;66:1157-64.

16. Kamat AM, Vlahou A, Taylor JA, Hudson ML, Pesch B, Ingersoll MA, et al. Considerations on the use of urine markers in the management of patients with high-grade non-muscleinvasive bladder cancer. Urol Oncol.2014;32:1069-77. 
17. Potretzke A, Hillman L, Wong K, Shi F, Brower R, Mai S et al. NLR is predictive of upstaging at the time of radical cystectomy for patients with urothelial carcinoma of the bladder. Urol Oncol.2014;32:631-6.

18. Mano R, Baniel J, Shoshany 0, Margel D, Bar-On T, Nativ 0 , et al. Neutrophil-to-lymphocyte ratio predicts progression and recurrence of non-muscle-invasive bladder cancer. Urol Oncol.2015;33:67.e1-7.

19. Hermanns T, Bhindi B, Wei Y, Yu J, Noon AP, Richard PO, et al. Pre-treatment neutrophil-to-lymphocyte ratio as predictor of adverse outcomes in patients undergoing radical cystectomy for urothelial carcinoma of the bladder. Br J Cancer.2014;111:444-51.

20. Gondo T, Nakashima J, Ohno Y, Choichiro O, Horiguchi $Y$, Namiki K, et al. Prognostic value of neutrophil-tolymphocyte ratio and establishment of novel preoperative risk stratification model in bladder cancer patients treated with radical cystectomy. Urology.2012;79:1085-91.
21. Wei $Y$, Jiang $Y Z$, Qian WH. Prognostic role of NLR in urinary cancers: a meta-analysis. PLoS One.2014;9:e92079.

22. Masson-Lecomte A, Rava M, Real FX, Hartmann A, Allory $Y$, Malats N. Inflammatory biomarkers and bladder cancer prognosis: a systematic review. Eur Urol.2014;66:1078-91.

Correspondence address:

Giorgio Ivan Russo, MD

Dipartimento di Urologia

Università degli Studi di Catania

Piazza Università, 2

95131 Catania

Fax: +39 95 378-2373

E-mail: giorgioivan@virgilio.it 